\title{
The Physical Activity Enjoyment Scale (Paces) as a Two-Dimensional Scale: Exploratory and Invariance Analysis
}

\author{
Filipe Rodrigues ${ }^{1,2,3}$, Pedro Forte ${ }^{3,4,5}$, Diogo Santos Teixeira ${ }^{6,7}$, Luís Cid ${ }^{1,3}$, Diogo Monteiro ${ }^{3,8}$ \\ Affiliations: 'Polytechnique Institute of Santarém, Sport Science School of Rio Maior (ESDRM-IPSantarém), Rio Maior, Portugal, ${ }^{2}$ Research Center in \\ Quality of Life (CIEQV), Santarém, Portugal, ${ }^{3}$ Research Center in Sport, Health and Human Development (CIDESD), Vila Real, Portugal, ${ }^{4}$ Instituto Superior

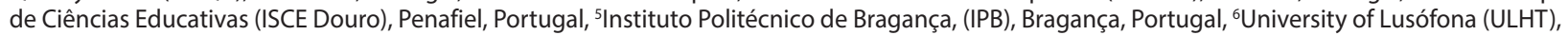 \\ Lisbon, Portugal, ${ }^{7}$ Center for the Study of Human Performance (CIPER), Lisbon, Portugal, ${ }^{8}$ Polytechnic of Leiria, ESECS, Leiria, Portugal
}

Correspondence: F. Rodrigues, Av. Dr. Mário Soares n¹10, 4020-413 Rio Maior, Portugal. E-mail: ptfiliperodrigues@gmail.com

\begin{abstract}
The current study aims to examine the Physical Activity Enjoyment Scale (PACES) as a two-dimensional scale assessing two correlated but distinct dimensions of enjoyment. In total, 277 individuals (female $=108$ ) aged between 18 and 53 years $(M=35.66 ; S D=7.42)$ participated in the study. The unidimensional model solution displayed good fit. However, the exploratory structural equation modelling specification considering two correlated but distinct dimensions of enjoyment showed a better fit. This study was the first attempt to examine the possible existence of two dimensions within the eight-item PACES measure. The distinct aspect of the current research is to emphasize the complex and constant process of instrument validation. Scales should be viewed as a continuous process, and future methodological procedures will increase our understanding of instrument examination with more innovative statistical approaches.
\end{abstract}

Keywords: enjoyment, exploratory analysis satisfaction, fun

$@$ MJSSMontenegro

PACES EXPLORATORY AND INVARIANCE ANALYSIS

http://mjssm.me/?sekcija=article\&artid=214

Cite this article: Rodrigues, F., Forte, P., Teixeira, D. S., Cid, L., \& Monteiro, D. (2021). The Physical Activity Enjoyment Scale (Paces) as a Two-Dimensional Scale: Exploratory and Invariance Analysis. Montenegrin Journal of Sports Science and Medicine, 10 (1), 61-66. doi: 10.26773/mjssm.210309

\section{Introduction}

People are emotional beings who relate to behaviours that are interesting and promote pleasure. In contrast, individuals avoid engaging in activities they do not like or have an associated negative meaning. Hence, it is expected that behaviours (e.g., physical activity) individuals like and are pleasurable will be pursued with greater engagement and commitment than activities they do not like (Jekauc \& Brand, 2017).
One theoretical outcome of self-determined motivation and predictor of intentions towards physical activity itself is enjoyment (Mullen et al., 2011). The experience of enjoyment reflects generalized feelings, such as pleasure, liking, and satisfaction (Moore Yin, et al., 2009) and has recently received great attention towards its significant association with several outcomes in different contexts, such as physical education (Gardner, et al., 2017), sport (Teixeira, et al., 2019), and exercise (Rodrigues, et al., 2020). 
Even though enjoyment has been assessed in several studies in recent years (Monteiro, et al., 2018; Mullen et al., 2011; Rodrigues, et al., 2019), no measures of a multi-dimensional structure have been considered for practical use. Instead, researchers have assumed enjoyment to be a global experience of liking and fun, a possible bias issue associated with the fact that there could be different dimensions of enjoyment individuals experience when exercising. As stated by several authors (Gråstén, et al., 2012; Gråstén \& Watt, 2017; Moreno, et al.; López, 2014), enjoyment could be defined as a multi-dimensional structure related to enthusiasm, excitement, and/or cognition. Therefore, this research intends to review the assessment of enjoyment when engaging in physical activity.

The enjoyment of physical activity is defined as a positive outcome to the movement experience that reflects feelings such as enthusiasm and excitement resulting from the activity itself (Raedeke, 2007). Thus, individuals who engage in exercise and sports for intrinsic motivations are more likely to experience higher levels of enjoyment (Monteiro et al., 2018; Rodrigues et al., 2020). Extending its importance, enjoyment can also be a predictor of behavioural outcomes, such as intentions towards leisure-time physical activity (Gardner, et al., 2017), commitment to sport (Granero-Gallegos, et al., 2017), and persistence in exercise (Rodrigues et al., 2018).

To date, several instruments have been developed and applied to measure enjoyment in physical activity. For example, Markland and Hardy (1993) have created the Exercise Motives Scale (EMI), encompassing four items measuring the experience of joy when exercising; Kendzierski and DeCarlo (1991) validated their Physical Activity Enjoyment Scale (PACES), which is an 18-item scale focused on assessing enjoyment only. In some studies, enjoyment has been assessed using single items (DiLorenzo, et al, 1998), representing little evidence of validity and reliability. Nevertheless, the most commonly used instrument nowadays is the eight-item version of PACES (Mullen et al., 2011), which advanced clinical assessment and reduced participants' burden. This shorter version has been translated and validated in several countries, including Portugal (Monteiro, et al., 2017; Teques, et al., 2017), Spain (Moreno et al., 2014), Germany (Jekauc, et al., 2013), and China (Chung \& Leung, 2018).

Little is known about the existence of different dimensions of enjoyment during physical activity. Looking at the PACES measure, the items could hold different types of enjoyment. For example, the item "It is very stimulating" could represent a more emotional representation or satisfaction on engaging in physical activity, whereas the item "It is a lot of fun" seems more relatable to fun and high arousal during training sessions. Kendzierski and DeCarlo (1991) have called for further analysis regarding PACES, suggesting that this scale could be broken down into components. Crooker, Marcel, and Gessaroli (1995) reinforced this statement, suggesting that the factor structure might not be unidimensional; rather, items seem to reflect different types of enjoyment. More recently, other authors (Gråstén \& Watt, 2017; Jekauc et al., 2013; Moreno et al., 2014) have suggested that enjoyment could have different sides, leading to different outcomes; therefore, researchers have called for more studies on physical activity enjoyment assessment.

Due to the widespread use of PACES, there is a need to rigorously evaluate the properties of the scale to determine a possible two-correlated factor structure that can be uniformly applied to future settings. Furthermore, a key concern currently limiting the expansion of the field of the assessment of physical activity enjoyment is the lack of exploratory measurement models for quantifying whether enjoyment is a global experience of liking something, or is it a multi-dimensional construct that could be associated with different outcomes (e.g., intention, engagement). This study explored PACES as a multi-dimensional measure of enjoyment. It is hypothesized that PACES could have a two-dimensional factor structure, assessing correlated but distinct constructs according to previous assumptions (Kendzierski \& DeCarlo, 1991). To confirm if the hypothesized two-correlated model would be equivalent between groups with different characteristics, measurement invariance analysis is performed between male and female. Based on previous assumptions, it is expected that the model would be equivalent between genders (Monteiro et al., 2017).

\section{Methods}

\section{Participants and procedures}

In total, 277 individuals (female $=108)$ aged between 18 and 53 years $(\mathrm{M}=35.66$; $\mathrm{SD}=7.42)$ participated in current study. This study was approved by the Ethical Committee (registration number: CE-UBI-pJ-2018-044:ID683); all procedures conducted in this research were in accordance with the 1964 Helsinki Declaration and its later amendments or comparable ethical standards. Then, sport club managers and gym executives were contacted, and objectives were explained. After obtaining approval, individuals were approached before training sessions and asked to participate voluntarily in this study. Participants who agreed signed informed consent prior to completing the questionnaire. Individuals took approximately 10 minutes to complete the scale.

\section{Measure}

Individuals completed the eight-item PACES version (Mullen et al., 2011). This scale comprises eight items measuring the degree of enjoyment individuals feel when exercising, to which participants respond to each item using a seven-point Likert scale anchored from 1 ("totally disagree") to 7 ("totally agree").

\section{Statistical analysis}

All procedures were performed using the Mplus 7.4 software (Muthén \& Muthén, 2010). We used the Robust Maximum Likelihood estimator since it is robust to the non-normality and non-independence of observations (Yuan \& Bentler, 2002). According to the theoretical hypothesis, we tested a unidimensional and a two-dimensional model specification. The unidimensional specification model was conducted to test if individuals perceive items as one factor only. A two-dimensional model was performed that considered the analysis of four specialists, each from different fields of scientific expertise (teacher, clinical psychologist, sport psychologist, and researcher in sport science). Next, a second evaluation panel composed of four other specialists evaluated the item distribution 
into two factors; together with the first panel of specialists, they came to a consensual judgment of the item distribution. In the unidimensional model, items were restricted to load only on their respective factor; whereas in Exploratory Structural Equation Modelling (ESEM) cross-loadings were targeted to be as close to zero as possible using the oblique target rotation procedure (Browne, 2001).

Chi-square statistics will be reported for visual orientation, but will not be considered to assess model fit since they are oversensitive on sample size and model complexity (Hair, et al., 2019). Therefore, this research considered traditional and incremental goodness-of-fit indexes: Comparative Fit Index (CFI), Tucker-Lewis Index (TLI), and Root Mean Square Error of Approximation (RMSEA). As cutoffs, values $\geq 0.90$ for CFI and TLI are typically interpreted to reflect acceptable fit (Byrne, 2016; Hair, et al., 2014; Marsh, et al., 2004). For RMSEA, values of $\leq 0.80$ were suggestive of reasonable fit (Marsh et al., 2004). Raykov's composite reliability coefficient (1997) was calculated for the subscale scores. Coefficient scores above or equal 0.70 provide acceptable internal consistency (Raykov, 1997).

To confirm if the hypothesized two-correlated model would be equivalent between groups with different charac- teristics, measurement invariance analysis was performed between males and females. First, the two-correlated model was tested in each sample. Then, for multigroup analysis, several levels of measurement invariance were measured according to Hair et al. (2019): configural invariance, weak factorial invariance, strong invariance, and strict factorial invariance. Model comparisons were made according to differences in CFI and TLI (Marsh et al., 2010). Differences below 0.01 were indicative of invariance moving ahead to the next level. Measurement invariance was achieved if all levels were below cutoffs, indicating that the model is equivalent between groups (Byrne, 2016).

\section{Results}

Results of the factor structure of the models are displayed in Table 1. The unidimensional model provided an acceptable fit to the data. Then, the two-correlated factor model was tested considering item distribution based on the evaluation panel described earlier. Items 1, 3, 6, and 7 were loaded into one specific factor, and items $2,4,5$, and 8 were loaded into another factor. The two-correlated model solution displayed excellent fit, suggesting the existence of two dimensions within the shorter eight-item measure.

Table 1. Model fit indexes

\begin{tabular}{lccccc}
\hline Model & $X^{2}$ & $\mathrm{df}$ & CFI & TLI & RMSEA \\
\hline Unidimensional factor CFA & 248.829 & 20 & .949 & .943 & .076 \\
Two-correlated factor ESEM & 120.865 & 13 & .981 & .959 & .065 \\
Two-correlated factor ESEM - female & 118.369 & 13 & .997 & .996 & .040 \\
Two-correlated factor ESEM - male & 117.478 & 13 & .997 & .987 & .042 \\
\hline
\end{tabular}

Note. $x^{2}=$ chi-square test; $\mathrm{df}=$ degrees of freedom; $\mathrm{CFI}=$ Comparative Fit Index; TLI = Tucker-Lewis Index; RMSEA $=$ Root Mean Squared Error of Approximation..

In the analysis of factor loadings (see Table 2), all items significantly loaded their respective factors in both unidimensional and two-correlated factor models. Several cross-loadings were found in the two-correlated factor specification; however, differences in the factor loading between target factor and non-target factor were below
0.15 , suggesting retaining the item in the factor in which it loaded the most. Composite reliability scores were above acceptable (>.70) in the unidimensional and two-correlated model specification, as seen in Table 2. In light of these results, the two-correlated factor model was retained for further analysis.

Table 2. Factor loadings of the original and the re-specified factor models

\begin{tabular}{ccccc}
\hline & \multicolumn{2}{c}{ Unidimensional model } & & \multicolumn{2}{c}{ Two-correlated factor model } \\
\cline { 2 - 2 } \cline { 5 - 5 } Item 1 & $.80^{* *}$ & & $.86^{* *}$ & .18 \\
Item 2 & $.81^{* *}$ & & $.31^{*}$ & $.75^{* *}$ \\
Item 3 & $.83^{* *}$ & & $.80^{*}$ & $.40^{*}$ \\
Item 4 & $.89^{* *}$ & & $49^{* *}$ & $.83^{* *}$ \\
Item 5 & $.87^{* *}$ & & $.22^{*}$ & $.79^{* *}$ \\
Item 6 & $.89^{* *}$ & & $.79^{* *}$ & $.25^{*}$ \\
Item 7 & $.84^{* *}$ & & $.82^{* *}$ & $.39^{*}$ \\
Item 8 & $.80^{* *}$ & & $.10^{* *}$ & $.74^{* *}$ \\
\hline CR & .95 & & .89 & .88 \\
\hline
\end{tabular}

Note. $\lambda=$ factor loadings; $F=$ Factor; target loadings are in bold; $C R=$ Composite Reliability; ${ }^{*} \mathrm{p}<0.05 ;{ }^{* *} \mathrm{p}<0.001$.

The two-correlated model was used to test measurement invariance between genders. The two-correlated model displayed acceptable fit in all samples, as seen in Table 3. Multi- group analysis shows that the measurement model was equivalent between samples and between gender since all invariance criteria were met $(\Delta \mathrm{CFI}<.01)$ and $(\Delta \mathrm{TLI}<.01)$. 
Table 3. Multigroup analysis of the two-correlated factor model between genders

\begin{tabular}{lcccccc}
\hline Model & $\mathrm{X}^{2}$ & $\mathrm{df}$ & $\mathrm{CFI}$ & $\Delta \mathrm{CFI}$ & $\mathrm{TLI}$ & $\Delta \mathrm{TLI}$ \\
\hline Configural Invariance & 206.559 & 52 & .998 & - & .997 & - \\
Weak Factorial Invariance & 198.449 & 50 & .998 & .001 & .998 & .001 \\
Strong Invariance & 139.184 & 54 & .999 & .001 & .999 & .002 \\
Strict Factorial Invariance & 128.177 & 48 & .999 & .001 & .999 & .002 \\
\hline
\end{tabular}

Note. $\Delta \mathrm{CFI}=$ differences in $\mathrm{CFI} ; \Delta \mathrm{TLI}=$ differences in $\mathrm{TLI}$.

\section{Discussion}

The aim of this research consisted of exploring the PACES as a two-dimensional scale assessing two correlated but distinct factors of enjoyment. The results reveal that the PACES could have two representations of enjoyment in individuals engaging in physical activity. Also, male and female physical activity participants experience both types of proposed enjoyment dimensions equally. The results will be discussed based on existing literature.

The first step consisted in examining a model considering the eight-item PACES as a one-factor measure. The CFA specification had an adequate fit, supporting previous studies using the same scale in the physical activity domains (Monteiro et al., 2017; Teques et al., 2017) and other health and academic contexts (Chung \& Leung, 2018; Moreno et al., 2014; Mullen et al., 2011). Thus, we moved forward on testing a possible two-dimensional factor model. Following examination of the hypothesis and evaluations made by the panel of specialists, items were loaded into two specific factors, and unintended items were forced to zero following assumptions of ESEM analysis. Items 1 (I find it pleasurable), 3 (It is very pleasant), 6 (It is very exhilarating), and 7 (It is very stimulating) were loaded on Factor 1. Items 2 (It is very refreshing), 4 (It is very invigorating), 5 (It is very gratifying), and 8 (It is a lot of fun) were loaded on Factor 2. The two-correlated model specification provided greater fit to the data (Hair et al., 2019) compared to the unidimensional model solution, showing possible existence of two dimensions of enjoyment.

Items loaded their respective factor significantly, showing no significant cross-loadings on unintended factors. These results support previous assumptions of a multi-dimensional PACES scale (Crooker et al., 1995; Kendzierski \& DeCarlo, 1991; Moreno et al., 2014), showing that the eight-item version could be suited in a two-correlated factor model. In this regard, to confirm if the hypothesized two-correlated model would be equivalent between groups with different characteristics, measurement invariance analysis was performed between males and females.

With respect to the measurement invariance analysis, the results support the equivalence of the two-correlated model version between gender since invariance levels were respected, as suggested by several authors (Byrne, 2017; Marsh et al., 2010). The invariance between groups indicates that the two-correlated factor model is being measured across groups, being interpreted in a conceptually similar manner by individuals representing different groups. Current findings showed that the two-correlated factor model is a reliable and valid measure on assessing two distinct factors of physical activity enjoyment in female and male individuals engaging in either sports or exercise. However, contrary to the results of Moore et al. (2009), in the present research, the instrument was invariant between genders. Differences could rely on the nature of physical activity and age. In this study, participants were physically active adults, whereas in the study conducted by Moore et al. (2009) the sample was composed of children in the physical education context. Nevertheless, the measurement model was equivalent between gender, showing its applicability in both male and female physical activity participants as displayed in previous research (Monteiro et al., 2017; Moore et al., 2009; Teques et al., 2017).

At a theoretical standpoint and considering the reports from the panel of specialists, defining the two dimensions of the PACES as "fun" and "satisfaction" is suggested. Fun (item $2,4,5$, and 8) could be defined as a pleasurable or amusement entertainment. In fact, Item 2 "I find it a lot of fun" reflects the particularity of recreational behaviour very well, being encountered in the physical activity domain. Thus, fun could be more pronounced when others are involved. That is, engaging in physical activity with peers, friends, and other important persons could increase the likelihood of experiencing fun (Monteiro et al., 2017). Also, when people engage in behaviour for fun, they seek the experience of pleasure and delight. The experience of having fun could thus be related to a state of flow (Csikszentmihalyi, 2013). When an individual is in the flow state, they are completely connected with the behaviour at hand, and without any conscious decision making, they lose awareness of time. As typically stated, "time flies when you are having fun".

Satisfaction (item 1, 3, 6 and 7) is at the core of the in-moment experience, reflecting the individual liking of physical activity, which results from personal interest towards the behaviour. Specifically, it is argued that satisfaction during physical activity is a satisfying feeling about the behaviour and willingness to continue pursuing it on the long-term (Rodrigues et al., 2019). Enjoyment should, therefore, be provided in every training session. Thus, arguably, enjoyment satisfaction would be a stronger predictor pf physical activity persistence, compared to fun. However, more studies are needed to explore this hypothesis based on current findings and previous studies in the sport (Teixeira et al., 2019) and exercise context (Rodrigues et al., 2020).

It is noteworthy that these are suggestions for defining these proposed factors, and more studies are warranted. Even though the measurement model displayed an existing two-factor structure, we encourage more studies to examine if the model would display similar results as those here reported. We intend to motivate a discussion and research about the possibility of the distinctiveness of enjoyment dimensions and the similarities of these types of enjoyment and their possible interrelationships. Moreover, future research will lead to new and powerful insights, albeit inevitably demonstrating contradictory results, which should be viewed as research enhancement rather than failure.

Some limitations should be addressed. We analysed the eight-item version and not the original eighteen-item proposed by Kendzierski and DeCarlo (1991). The original ver- 
sion could represent more dimensions of enjoyment during physical activity. Crooker et al. (1995) suggested that the factor structure might not be unidimensional; rather, items seem to identify the antecedents and consequences of enjoyment. In this regard, more research is needed on the assessment of physical activity enjoyment. Finally, longitudinal measurement seems paramount, since enjoyment may fluctuate over time (Chung \& Leung, 2018).

\section{Conclusion}

The current study filled a gap to allow future research to examine a possible multi-dimensional measure of the PACES (Kendzierski \& DeCarlo, 1991). The two-correlated version presented reliable and validated results in the physical activity context. This instrument seems to measure two dimensions of enjoyment when engaging in training sessions. Assessing enjoyment increases health, exercise, and sport professionals' insight into how to promote physical activity. Since it presents an outcome of intrinsic motivation, as well as a predictor of several cognitive and behavioural outcomes, enjoyment should be constantly measured as a way to understand how individuals are experiencing satisfaction and fun during physical activity. The distinctiveness of current research is to emphasize the complex and constant process of instrument validation to advance theoretical assumptions. Scales should be viewed as a continuous process, and future methodological procedures will increase our understanding of instrument examination with more innovative statistical approaches..

\section{Acknowledgement}

The first author was supported by the national funds through the Portuguese Foundation for Science and Technology, I.P., under the project UIDP/04748/2020, and the last two authors were supported under the project UID04045/2020.

\section{References}

Browne, M. (2001). An overview of analytic rotation in exploratory factor analysis, Multivariate Behavioral Research, 36 (1), 111-150.

Byrne, B. (2016). Structural equation modeling with AMOS. Basic concepts, applications, and programming (3rd ed.): New York: Taylor \& Francis Group, LLC.

Chung, P. K., \& Leung, K. M. (2018). Psychometric Properties of Eight-Item Physical Activity Enjoyment Scale in a Chinese Population. Journal of Aging \& Physical Activity, 1-6. doi: 10.1123/japa.2017-0212

Crooker, P., Marcel, B., \& Gessaroli, M. (1995). Measuring enjoyment in youth sport settings: A confirmatory factor analysis of the Physical Activity Enjoyment Scale. Journal of Sport \& Exercise Psychology, 17(2), 200-205. doi: 10.1123/jsep.17.2.200

DiLorenzo, T., Stucky-Ropp, R., Vander Wal, J., \& Gotham, H. (1998). Determinants of exercise among children. II. A longitudinal analysis. Preventive Medicine, 27(3), 470-477. doi: 10.1006/pmed.1998.0307

Gardner, L., Magee, C., \& Vella, S. (2017). Enjoyment and Behavioral Intention Predict Organized Youth Sport Participation and Dropout. Journal of Physical Activity \& Health, 14(11), 861-865. doi: 10.1123/jpah.2016-0572

Granero-Gallegos, A., Gomez-Lopez, M., Rodriguez-Suarez, N., Abraldes, J., Alesi, M., \& Bianco, A. (2017). Importance of the Motivational Climate in Goal, Enjoyment, and the Causes of Success in Handball Players. Frontiers in Psychology, 8, 2081. doi: 10.3389/fpsyg.2017.02081

Gråstén, A., \& Watt, A. (2017). A motivational model of physical education and links to enjoyment, knowledge, performance, total physical activity and body mas index. Journal of Sport Science \& Medicine, 16(318-327).

Gråstén, A., Jaakola, T., Liukkonen, J., Watt, A., \& Yli-Piipari, S. (2012). Prediction of Enjoyment in School Physical Education. Journal of Sport Science and Medicine, 11(2), 260-269.

Hair, J., Black, W., Babin, B., \& Anderson, R. (2019). Multivariate Data Analysis (8th ed.): New Jersey: Pearson Educational, Inc.

Jekauc, D., \& Brand, R. (2017). Editorial: How do Emotions and Feelings Regulate Physical Activity? Frontiers in Psychology, 8, 1145. doi: 10.3389/fpsyg.2017.01145

Jekauc, D., Voelkle, M., Wagner, M. O., Mewes, N., \& Woll, A. (2013). Reliability, validity, and measurement invariance of the German version of the physical activity enjoyment scale. Journal of Pediatric Psychology, 38(1), 104-115. doi: 10.1093/jpepsy/jss088

Kendzierski, D., \& DeCarlo, K. (1991). Physical Activity Enjoyment Scale: Two Validation Studies. Journal of Sport \& Exercise Psychology, 13(1), 50-64. doi: 10.1123/ jsep.13.1.50

Markland, D., \& Hardy, L. (1993). The Exercise Motivations Inventory: preliminary development and validity of a measure of individuals' reasons for participation in regular physical exercise. Personality \& Individual Differences, 15(3), 286-296. doi: 10.1016/0191-8869(93)90219-S

Marsh, H., Hau, K., \& Wen, Z. (2004). In search of Golden Rules: comment on hypothesis-testing approaches to setting cutoff values for fit indexes and dangers in overgeneralizing $\mathrm{Hu}$ and Bentler's (1999) Findings. Structural Equation Modeling: A Multidisciplinary Journal, 11(3), 320-341. doi: 10.1207/s15328007sem1103_2

Monteiro, D., Nunes, G., Marinho, D., Couto, N., Antunes, R., Moutão, J., \& Cid, L. (2017). Translation and adaptation of the physical activity enjoyment scale (PACES) in a sample of Portuguese athletes, invariance across genders nature sports and swimming. Brazilian Journal of Kinanthropometry \& Human Performance, 19(6), 631-643. doi: 10.5007/1980-0037.2017v19n6p631

Monteiro, D., Pelletier, L., Moutão, J., \& Cid, L. (2018). Examining the motivational determinants of enjoyment and the intention to continue of persistent competitive swimmers. International Journal of Sport Psychology, 49(6), 484.504. doi: 10.7352/IJSP.2018.49.484

Moore, J., Yin, Z., Hanes, J., Duda, J., Gutin, B., \& Barbeau, P. (2009). Measuring Enjoyment of Physical Activity in Children: Validation of the Physical Activity Enjoyment Scale. Journal of Applied Sport Psychology, 21(S1), S116-S129. doi: 10.1080/10413200802593612

Moreno, J.-A., González-Cutre, D., Martínez, C., Alonso, N., \& López, M. (2014). Propiedades psicométricas de la Physical Activity Enjoyment Scale (PACES) en el contexto español. Estudios de Psicología, 29(2), 173-180. doi: 10.1174/021093908784485093

Mullen, S., Olson, E., Phillips, S., DSzabo, A., Wójcicki, T., Mailey, E., ... McAuley, E. (2011). Measuring enjoyment of physical activity in older adults: invariance of the physical activity enjoyment scale (paces) across groups and time. 
International Journal of Behavioral Nutrition \& Physical Activity, 8, 103. doi: 10.1186/1479-5868-8-103

Muthén, L., \& Muthén, B. (2010). Mplus User's Guide. Muthén \& Muthén: Los Angeles.

Raedeke, T. D. (2007). The Relationship Between Enjoyment and Affective Responses to Exercise. Journal of Applied Sport Psychology, 19(1), 105-115. doi: $10.1080 / 10413200601113638$

Raykov, T. (1997). Estimation of Composite Reliability for congeneric measures. Applied Psychological Measurement, 21(2), 173-184. doi: 10.1177/01466216970212006

Rodrigues, F., Bento, T., Cid, L., Pereira Neiva, H., Teixeira, D., Moutao, J., Marinho, D., Monteiro, D. (2018). Can Interpersonal Behavior Influence the Persistence and Adherence to Physical Exercise Practice in Adults? A Systematic Review. Frontiers in Psychology, 9, 2141. doi: 10.3389/fpsyg.2018.02141

Rodrigues, F., Teixeira, D. S., Neiva, H., Cid, L., \& Monteiro, D. (2020). The bright and dark sides of motivation as predictors of enjoyment, intention, and exercise persistence. Scandinavian Journal of Medicine \& Science in Sports, 30(4), 787-800. https://doi.org/10.1111/sms.13617

Rodrigues, F., Teixeira, D., Cid, L., \& Monteiro, D. (2019). Have you been exercising lately? Testing the role of past behavior on exercise adherence. Journal of Health Psychology, 1359105319878243. doi: 10.1177/1359105319878243

Teixeira, D. S., Pelletier, L. G., Monteiro, D., Rodrigues, F., Moutao, J., Marinho, D., \& Cid, L. (2019). Motivational patterns in persistent swimmers: A serial mediation analysis. European Journal of Sports Science. doi: 10.1080/17461391.2019.1675768

Teques, P., Calmeiro, L., Silva, C., \& Borrego, C. (2017). Validation and adaptation of the Physical Activity Enjoyment Scale (PACES) in fitness group exercisers. Journal of Sport \& Health Science. doi: 10.1016/j. jshs.2017.09.010

Yuan, K., \& Bentler, P. (2002). Structural Equation Modeling with Robust Covariances. Sociological Methodology, 28(1). doi: 10.1111/0081-1750.00052 